Proceedings of the 10th International Ruminant Reproduction Symposium (IRRS 2018); Foz do Iguaçu, PR, Brazil, September 16th to 20th, 2018.

\title{
Applications of large-scale molecular profiling techniques to the study of the corpus luteum
}

\author{
Joy L. Pate*, Camilla K. Hughes
}

Penn State University, Center for Reproductive Biology and Health, Department of Animal Science, University Park, PA 16802 USA.

\begin{abstract}
The corpus luteum (CL) is vital for the establishment and maintenance of pregnancy. Throughout the history of luteal biology, cutting-edge technologies have been used to develop a thorough understanding of the functions of specific luteal cell types, the signaling pathways that result in luteal cell stimulation or demise, and the molecules that regulate specific functions of luteal cells. The advent of largescale profiling technologies such as transcriptomics, proteomics, and metabolomics, has brought with it an interest in discovering novel regulatory molecules that may provide targets for manipulation of luteal function or lifespan. Although the work to date is limited, transcriptomics have been effectively used to provide a global picture of changes in mRNA that relate to luteal development, steroidogenesis, luteolysis or luteal rescue. Some studies have been reported that profile microRNA (miRNA) and proteins, and although not yet published, metabolomics analyses of the CL have been undertaken. Thus far, these profiling studies seem to largely confirm earlier findings using targeted approaches, although previously unstudied molecules have also come to light as important luteal regulators. These molecules can then be studied using traditional mechanistic techniques. Use of profiling technologies has presented physiologists with unique challenges associated with analyses of big data sets. An appropriate technique for balancing the risks associated with type I (false discoveries) and type II (overlooking a real change) statistical error has not yet been developed and many big data studies may have potentially important differences that are overlooked. Also, it is imperative that attempts be made to integrate information from the various -omics studies before drawing conclusions based on expression of only one class of molecule, to better reflect the interdependency of molecular networks in cells. Currently, few analysis programs exist for such integrations. Despite challenges associated with these techniques, they have already provided new information about the biology of the CL, notably allowing identification of a key regulator of acquisition of luteolytic capacity and providing a big-picture view of the subtle changes that occur in the CL during early pregnancy. As these technologies become more accurate and less expensive, and as analysis becomes more userfriendly, their use will become much more widespread and many new discoveries will be made. This review will focus only on relevant studies in which these technologies were used to study the CL of ruminants.
\end{abstract}

Keywords: bovine, corpus luteum, molecular profiling.

\section{Introduction}

In the 1600's, Regnier de Graaf described his observation of transient yellow globules that form from emptied ovarian follicles after coitus, noting that the number of globules was the same as the number of fetuses (Jocelyn and Setchell, 1972), and Marcello Malpighi first called this structure a corpus luteum, latin for yellow body. The function of the corpus luteum (CL) remained a mystery for 300 years, when experimental evidence was obtained that the CL was necessary for the maintenance of pregnancy (Simmer 1971; Frobenius 1999). This was followed by the discovery of the primary secretory product of the CL, progesterone, in the 1930's. Despite a slow beginning to the understanding of the function of the CL, once it was identified that this small structure was absolutely essential for the establishment and maintenance of pregnancy in all mammals, it captured the attention of reproductive biologists, and none more so than those interested in reproduction of domestic ruminants. Thus, in the last 50 years, great advances have been made in understanding luteal function, much of which came from studies in cows and sheep. While the goal of this research was to enhance fertility of these species, the knowledge of the basic biology of the CL could be generally applied to nonruminants, and because of its ephemeral nature, the CL has served as a model for many aspects of cellular biology, including angiogenesis, tumor development, steroidogenesis, roles of tissue-resident immune cells, and pathways of cellular death.

During the mid- to late 20th century, the hormonal regulators, second messenger molecules and biochemical reactions in steroidogenesis were elucidated. Sources of cholesterol as substrate for progesterone synthesis and intracellular signaling pathways were defined. Refined procedures to separate cells based on size led to a race to determine the origins and distinct functions of the small and large steroidogenic cells, and the discovery that oxytocin is produced in the CL prompted a flurry of research to determine if luteal oxytocin is necessary for uterine prostaglandin (PG)F2A release during luteolysis. The cellular heterogeneity that characterizes the $\mathrm{CL}$ also intrigued researchers, whose work revealed the contributions of endothelial cells, fibroblasts, pericytes and immune cells to development, function, and regression of the CL. The advent of technologies for identifying and quantifying steady state concentrations of mRNA in cells and tissue, including northern blotting, PCR, and qPCR, brought about a revolution in targeted-approach experimentation to elucidate how 
changes in luteal functions are driven by changes in gene expression. For more information about these discoveries and the general biology of the CL, the reader is referred to a number of reviews on ruminant luteal function (Niswender et al., 2000; Pate et al., 2012; Wiltbank et al., 2012; Miyamoto et al., 2013; Smith and Meidan, 2014).

\section{Transcriptomic profiling in the corpus luteum}

Using the technologies mentioned above, studies of mRNA concentrations in the CL have been hypothesis-driven, searching for the key changes in mRNA relative to receptor activation, signal transduction, steroidogenesis, cytokine production, and cell death pathways. Much has been learned about which pathways and genes were regulated during development and regression of the CL using this type of approach. However, more recently, researchers have used high throughput technologies to profile many (microarray) or all (sequencing) of the transcripts present in the CL from selected times or physiological states. This approach was at first criticized as being a fishing expedition, but identification of potentially important molecules that led to new hypotheses about luteal regulation has enhanced acceptance of these powerful approaches. Transcriptomic analyses have largely confirmed our understanding of luteal functions as determined by more targeted approaches, lending further support to previously drawn conclusions. Perhaps more importantly, they have also shed light on unexplored or potentially new cellular pathways and functions.

Development of the ruminant $\mathrm{CL}$ involves differentiation of follicular steroidogenic cells and it was suggested from cell-labeling studies that the small luteal steroidogenic cells (SLC) are derived from the thecal cells of the follicle, whereas the large cells (LLC) originally differentiate from granulosal cells (Alila and Hansel, 1984). Romereim et al. (2017) used microarrays to profile the transcriptomes of isolated granulosal, thecal, and separated luteal cells. This approach supported the existing model of differences between SLC and LLC, including identification of the LHCGR in greater abundance on SLC and the PTGFR in greater abundance on LLC. Additionally, it allowed for identification of six novel cell lineage markers each for the thecal cell-SLC lineage and the granulosal cell-LLC lineage. These lineage markers include molecules involved in ion and molecular transport and lysosomal function in LLC and are primarily molecules involved in signaling in SLC. Further, the transcriptome of the large steroidogenic cells indicated that these cells likely function in recruitment of immune and endothelial cells, activities that had not previously been ascribed to a particular luteal steroidogenic cell type. Baddela et al. (2018) reported 1276 differentially abundant mRNA in small and large luteal cells. The small luteal cells were enriched in mRNA responsible for immune cell recruitment, whereas the profile of large luteal cell mRNA suggested functions in regulating folliculogenesis, luteolysis, and small molecule metabolism. The reported purity of the separated cell populations was similar in these two studies, so the clear discrepancy between them may be due to the cell-type comparisons made. Baddela et al. used days 11-12 CL $(n=4)$ from timed estrous cycles. The stage of the cycle from which CL $(n=3)$ were collected in the study of Romereim et al. was not described. Because differentiation of the small and large cells is a somewhat continuous process, it is possible that functions associated with small and large cells are stagedependent. Although it remains to be determined which steroidogenic cell type is responsible for recruiting immune cells, other differentially abundant mRNA and predicted functions of small and large cells were fairly consistent between the two studies.

Differentiation and maximal steroidogenic capacity of the ruminant CL is dependent on luteinizing hormone. As might be expected, gonadotropic stimulation of the CL resulted in upregulation of genes related to lipid metabolism, cholesterol metabolism and progesterone production (Fatima et al., 2012). The most upregulated mRNA was fatty acid binding protein 5 (FABP5), which can transport lipids within cells to lipid droplets and mitochondria. To our knowledge, this potentially important regulator of steroidogenesis has not been studied in the CL. Transcriptomic analysis of day 4 and day 11 bovine CL also indicated that steroidogenic and cholesterol biosynthetic genes are upregulated in the midcycle CL, along with genes involved in immune response, whereas the day $4 \mathrm{CL}$ is characterized by genes related to cell cycle, DNA replication and metabolic processes (Kfir et al., 2018). This analysis also revealed that the developing CL expresses angiogenesis-promoting genes, whereas the mature CL expressed genes related to cessation of blood vessel sprouting.

As the CL develops, it must gain the capacity to regress in response to PGF2A (Tsai and Wiltbank, 1998). The inability of the developing CL to regress, despite clear responses to PGF2A, has intrigued ovarian biologists for decades, leading to comparison of the transcriptomes of early (day 4) and midcycle (dday 10) $\mathrm{CL}$ in response to exogenous PGF2A. Using microarrays, Goravanahally et al. (2009) found 167 differentially expressed genes, most of which were upregulated in the day $10 \mathrm{CL}$, likely reflecting differentiation and development of pathways for maximal steroidogenesis. This group then focused on the 20 genes that were associated with cell signaling pathways, as these genes could be regulators of luteal responsiveness to PGF2A. Collection of CL $24 \mathrm{~h}$ after a luteolytic injection of PGF2A showed upregulation of CAMKK2 in day 9, but not day 4, CL. Although the CL in this study were collected $24 \mathrm{~h}$ after the PGF2A injection, when luteolysis is advanced, this research group later showed that CAMKK2 is indeed a component of the PGF2A signaling pathway in day 10 , but not day 4, CL (Bowdridge et al., 2015), providing a good example of how a profiling experiment led to the discovery of a molecule that could potentially be targeted to regulate luteal function. Differences in PGF2A regulation of gene transcription were further 
delineated by Mondal et al (2011) who also used microarrays to determine differentially abundant genes in early (day 4) and midcycle (day 11) CL collected following administration of PGF2A. Prostaglandinregulated genes were detected in both types of CL, but the response was much more robust in the midcycle CL. Genes that were upregulated by PGF2A in day 11 , but not day 4, CL indicated activation of biological processes involved in receptor activity, cellular death, and immune cell-related genes, and many were genes that are under the control of ETS family transcription factors. This is a large family of transcription factors that is associated with regulation of many cellular functions, including apoptosis. Of note, almost all of the listed biological processes upregulated by PGF2A within $4 \mathrm{~h}$ in the midcycle, but not day $4 \mathrm{CL}$, include genes normally associated with an immune response, such as cytokines, chemokines and adhesion molecules. A macrophage marker, CD14, was upregulated by PGF2A on day 11 , but not day 4 , suggesting differential recruitment of immune cells into the mature $\mathrm{CL}$, after induction of luteal regression. The profiling study of Mondal et al. (2011) also led to further studies that defined the response of angiogenic factors to PGF2A in day 4 and day $11 \mathrm{CL}$ and their functional roles on luteal endothelial cells (Zalman et al., 2012). Overall, these studies support the earlier work from Wiltbank's group (Tsai and Wiltbank, 1998) showing that the day $4 \mathrm{CL}$ is not unresponsive to PGF2A, rather the responses of the day $4 \mathrm{CL}$ differ from those of an older CL that has acquired luteolytic capacity.

Shah et al. (2014) and Talbott et al. (2017) used microarrays to investigate temporal changes in gene expression during luteal regression, using water buffalo and cows, respectively. As in previous studies, changes in transcripts related to steroidogenesis, $\mathrm{LH}$ receptor signaling, and apoptosis were observed. Talbott et al. (2017) also confirmed earlier studies in which progesterone declined prior to any decrease in transcripts related to steroidogenesis, and in which luteolysis was characterized by changes in transcripts related to cholesterol availability. However, most of these transcripts also changed coincident to, not before, the decrease in progesterone. Transcription factor mRNA and transcripts indicating activation of cytokine signaling were altered prior to the decrease in progesterone, further supporting the growing evidence that inflammatory-like events are key mediators of PGF2A-induced luteolysis (Mondal et al., 2011; Atli et al., 2012; Shah et al., 2014; Talbott et al., 2017). Shah et al. also reported downregulation of CYP19A1 and differential abundance of estrogen-responsive genes, a novel finding. Although estrogen synthesis by the bovine $\mathrm{CL}$ is low, a role of intraluteal estrogen and estrogen receptor signaling in PGF2A-induced luteolysis was proposed. This would extend previous findings that estrogen can induce premature luteolysis (Wiltbank et al., 1961) and that follicular estradiol may be necessary for timing normal luteolysis (Villa-Godoy et al., 1985).

Atli et al. (2012) developed a model of repeated intrauterine infusions of physiological concentrations of PGF2A, coupled with luteal biopsies, to evaluate temporal changes in the CL during luteal regression. This study, which used qPCR to profile transcripts, indicated that activation of genes related to immune response and prostaglandin metabolism were necessary to ensure the progression of luteolysis. This model was further used to determine if PGE2, which is thought to be involved in luteal rescue during early pregnancy, could suppress PGF2A-induced gene expression. The magnitude of effect of PGF2A pulses on gene expression in the CL, as assessed using RNAseq, was quite large (Ochoa et al., 2018). Compared to saline infused controls, 572 mRNA were altered by PGF2A, with an additional 373 mRNA that differed from PGE2 and PGF2A + PGE2 infusions. Transcripts most significantly regulated by PGF2A included those associated with steroidogenesis, apoptosis, and signal transduction, as expected. These data also indicated that ceramide signaling may be associated with luteolysis. Remarkably, compared to saline controls, there were no differentially abundant mRNA in the CL following intrauterine infusions of PGE2 or PGE2 + PGF2A, and these CL did not regress, demonstrating that PGE2 can completely prevent PGF2A-induced changes in mRNA that would ensure luteolysis. A summary of the pathways associated with the stages of luteal development, maintenance and regression, as determined by RNA profiling of luteal tissue, is depicted in Fig. 1.

Little is known about changes that occur within the CL to facilitate its rescue during early pregnancy. When the CL of pregnancy was compared to midcycle (days 10-12) CL, differentially abundant mRNA gradually increased throughout pregnancy (Sakumoto et al., 2015), indicating that, once rescued, the CL is not static, but is actively regulated by either intrinsic or extrinsic factors that alter mRNA abundance to facilitate luteal survival and progesterone production. In this study, large changes in chemokine mRNA in the CL of pregnancy were noted, particularly a morethan 10-fold decrease in lymphotactin, a chemokine that recruits $\mathrm{T}$ cells, and a more-than 100 -fold increase in eotaxin, a chemokine that recruits eosinophils. This study also noted a more modest increase in growth factor-related mRNA during early pregnancy (Sakumoto et al., 2015). Similarly, a microarray study performed by Romero et al. (2013) demonstrated that in the ovine CL of early pregnancy, there is stabilization or upregulation of pathways related to interferon and cytokine signaling, cell-cell adhesion, and cytoskeleton, as compared to the late and regressing CL. Pentraxin-3, which is produced by several immune cell types, was stabilized in early pregnancy, but reduced during luteal regression. The authors suggest that this molecule may increase cellular resistance to stress (Romero et al., 2013). Overall, these and other studies demonstrate that chemokines and cytokines appear to be key regulators of both luteal regression and luteal survival during pregnancy. 


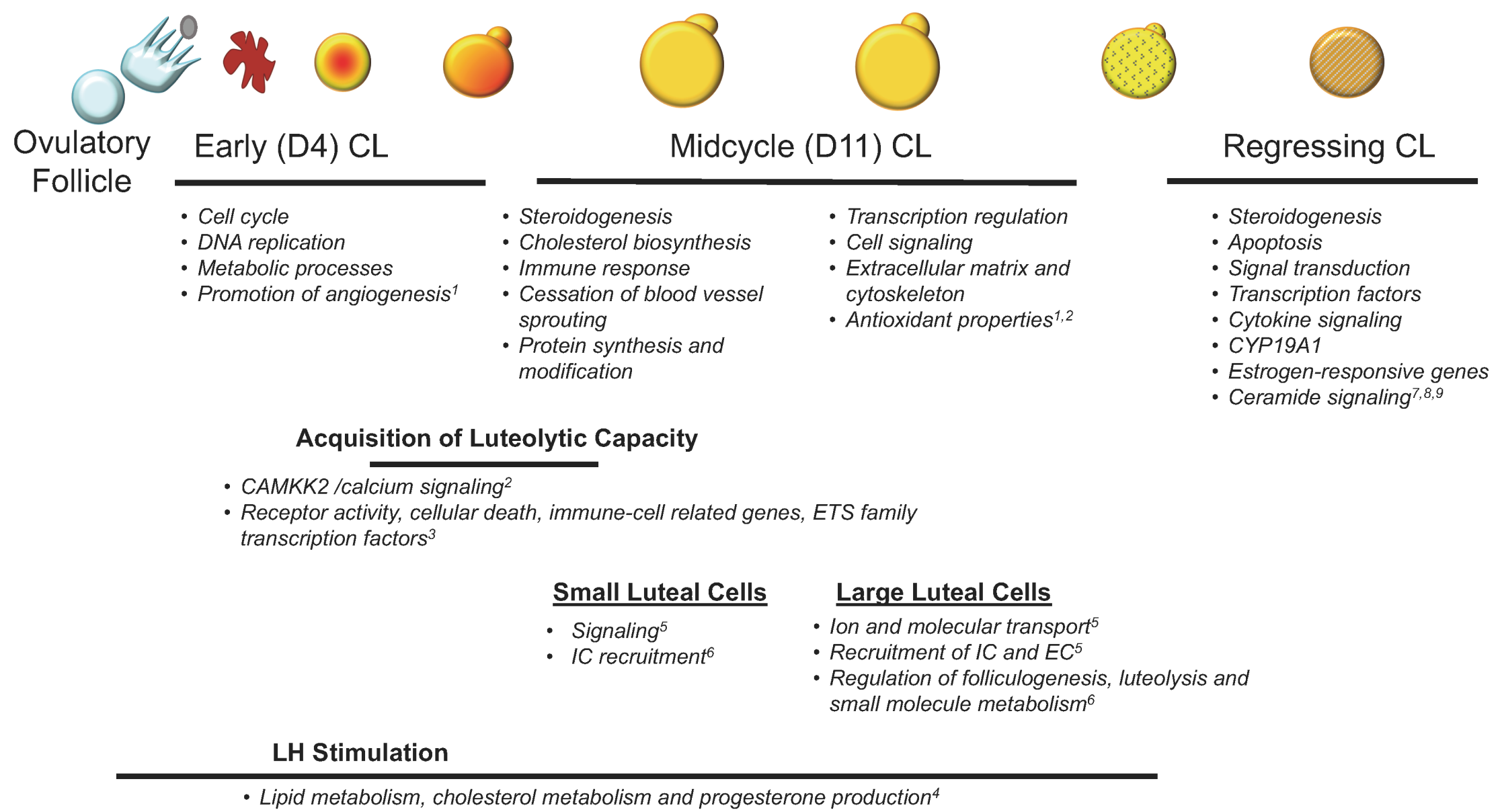

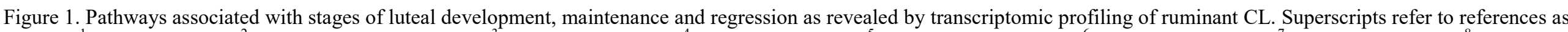

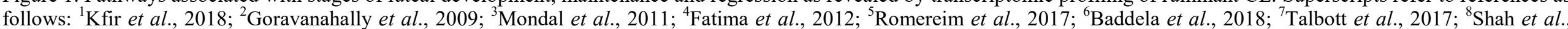
2014; ${ }^{9}$ Ochoa et al., 2018. 
The advent of RNAseq provided more sensitive and more accurate detection of mRNA, allowing for more comprehensive studies of the luteal transcriptome, and providing the opportunity to reveal potentially important transcripts that were previously unrecognized. In a recent RNAseq study comparing transcript abundance in bovine CL of day 17 of the estrous cycle and day 17 of pregnancy, 144 differentially abundant mRNA were reported and immune signaling pathways were among those predicted to be modulated in early pregnancy, as well as novel potential regulators of luteal rescue, including PPAR signaling and PDGF signaling (Hughes et al., 2018; Penn State University, Center for Reproductive Biology and Health, University Park, PA USA; unpublished data) Moore et al. (2016) used RNAseq to determine if CL and endometria of cattle of low or high genetic merit for fertility contained differentially abundant mRNA. Only 9 mRNA were different in the endometrium, whereas 560 mRNA were different in the CL, suggesting an important relationship between luteal function and fertility. Of the $560 \mathrm{DE}$ mRNA, $85 \%$ were lesser in abundance in the CL from low fertility cows, indicating a general reduction in luteal activity. These included genes related to steriodogenesis, extracellular matrix and RNA replication, indicating compromised luteal development and steroidogenic capacity. Conversely, mRNA related to PGF2A response were greater in CL from low fertility cows.
Although a primary focus in statistical analysis of transcriptomic datasets has been on the reduction of type I error, due to the large number of statistical tests performed, this problem has been largely corrected by the Benjamini Hochberg false discovery rate correction, which allows a researcher to choose a threshold (typically between 5 and 15\%) of false discoveries that they are willing to tolerate (Benjamini and Hochberg, 1995). However, while this method reduces type I error, it increases type II error and, in some studies, may cause researchers to overlook many genes, proteins, or metabolites that change in a biologically relevant way (Mudge et al., 2017). Recent experiments have demonstrated that in an RNAseq study of the CL of the estrous cycle and pregnancy, mRNA that were well below the false discovery rate cutoff were still differentially expressed $(\mathrm{P}<0.05)$ by two-fold or more when measured by qPCR (Hughes et al., 2018; Penn State University, Center for Reproductive Biology and Health, University Park, PA USA; unpublished data; Fig. 2). Given the cost and time associated with gene expression profiling experiments, the amount of information lost due to this increased type II error is concerning. One proposed solution to this problem is an optimized Pvalue cutoff, based on power and relative cost of type I and type II error, for each big data study (Mudge et al., 2017).

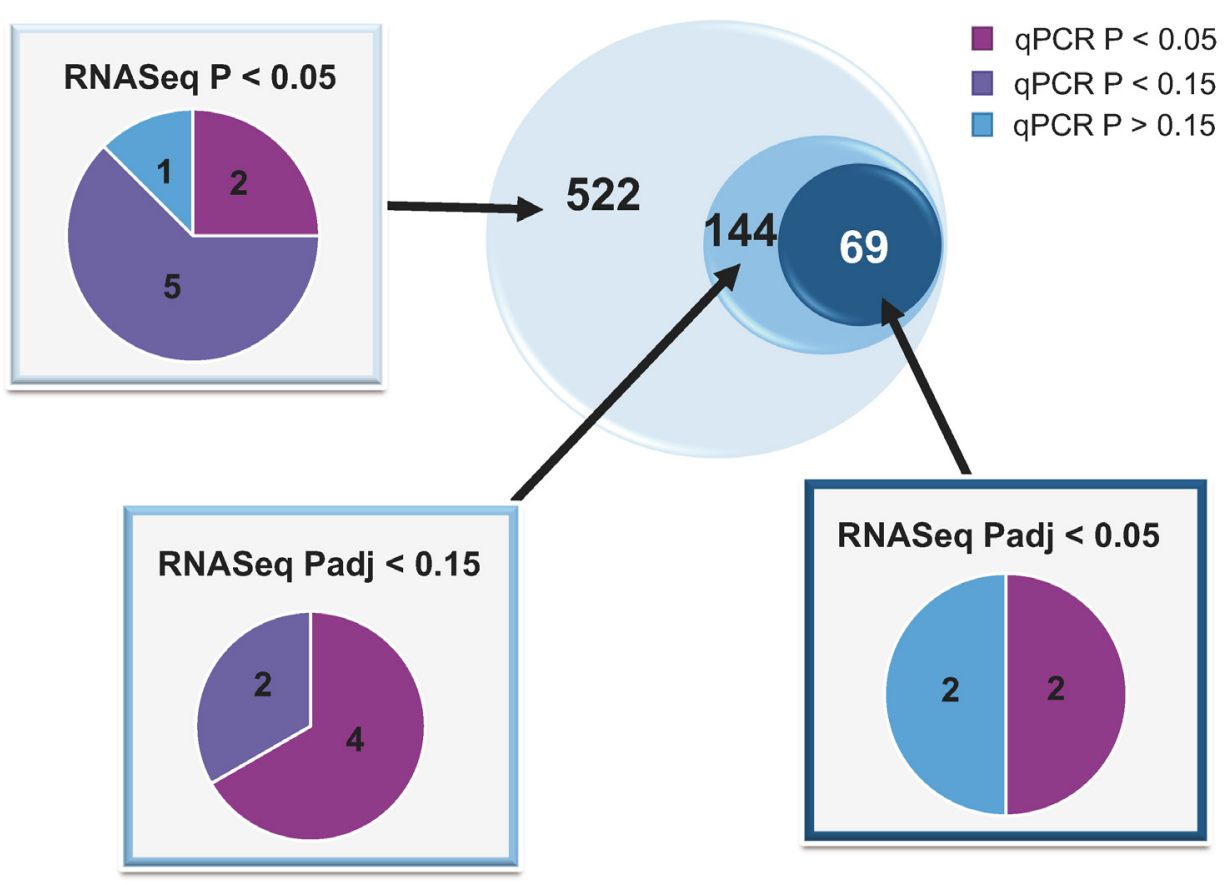

Figure 2. mRNA that were differentially abundant in a transcriptomics study (Hughes et al., 2018; Penn State University, Center for Reproductive Biology and Health, University Park, PA USA; unpublished data). Three P-value cutoffs were used $(\mathrm{P}<0.05$, padj $<0.15$, padj $<0.05$ ), with 522, 144, and 69 mRNA in each group. Padj-values are $\mathrm{P}$-values that have been adjusted for false discovery rate of $5 \%$ false discoveries. These three groups are represented by the three concentric circles. A subset of mRNA from each group was analyzed using qPCR $(\mathrm{n}=6)$; total number of mRNA analyzed by qPCR in each subset is represented within each pie chart as significantly $(\mathrm{P}<0.05)$, or with a tendency to be $(\mathrm{P}<0.15)$, differentially expressed, or not $\mathrm{DE}(\mathrm{P}>0.15)$. 


\section{MicroRNA profiling and regulation of the corpus luteum}

MicroRNA are single-stranded noncoding RNA, approximately $22 \mathrm{nt}$ in length, that serve as posttranscriptional regulators of gene expression. Following transcription, the precursor forms of miRNA undergo nuclear and cytoplasmic cleavage to form the mature miRNA. The mature miRNA binds to Argonaute 2 (AGO2), is then incorporated into the RNA-Induced silencing Complex (RISC), and the complex is targeted to the 3'UTR of mRNA with sufficient complementarity to the miRNA. This results in loss of mRNA translation to protein, either via translational repression or degradation of the mRNA. Individual miRNA can have numerous mRNA targets, and individual mRNA can be targeted by many miRNA, which makes elucidation of miRNAregulated signaling pathways quite complex. MicroRNA expression and activity can be both tissue- and stagespecific, and in some cases can even enhance, rather than suppress, synthesis of specific proteins. The complexity of miRNA biology necessitates confirmation of a proposed regulatory role in a particular tissue at a specific developmental or functional stage. For information on microRNA biology, the reader is referred to reviews by $\mathrm{He}$ and Hannon (2004), Treiber et al. (2012), Catalanotto et al. (2016), Maalouf et al. (2016a), and Tesfaye et al. (2018).

MicroRNA have captured the attention of biologists in many disciplines, but in particular, seem to have improved the understanding of the dynamic nature of reproductive tissues. Most studies of ovarian miRNA have focused on follicles and oocytes of nonruminant species (reviews: Christenson, 2010; Hossain et al., 2012; Li et al., 2015; McGinnis et al., 2015; Maalouf et al., 2016a). Hossain et al. (2009) cloned and sequenced a small RNA library derived from bovine ovary and found that some miRNA were dissimilar in abundance among cortex, follicles and CL. Using a screened target set of 115 genes likely to be regulated by abundant ovarian miRNA, pathway analysis indicated that the selected miRNA and their predicted targets were indeed involved in functions indicative of the dynamic nature of ovarian components, such as growth factor signaling, cellular growth and development, and cellular death.

Expression of miRNA in the CL may be developmentally regulated, because greater abundance of miRNA in the mature than in the developing CL has been reported (Maalouf et al., 2016b; Baddela et al., 2017), and the functions regulated by luteal miRNA shift from cellular metabolism and growth in the day 4 CL to cell cycle, cell death, and gene expression in the midcycle CL (Maalouf et al, 2016b). One of the upregulated miRNA, miR34a, targeted NOTCH1 and YY1 and promoted luteal cell progesterone production while suppressing proliferation of luteal cells (Maalouf et al., 2016b), consistent with a role in inhibition of growth while enhancing differentiated function. McBride et al. (2012) reported that 9 miRNA decreased and 8 increased during the follicular-luteal transition in sheep, and their predicted targets are involved in cellular development, differentiation, proliferation and survival. The difference in number and direction of DE
miRNA between these two studies is likely due to method of detection (microarray vs. Sanger sequencing) and comparison of mature CL to follicular (McBride et al., 2012) vs. immature luteal (Maalouf et al., 2016b) cells. When water buffalo CL of 3 estrous cycle stages were compared to granulosal cells using miRNAseq, more miRNA were CL-specific than granulosal cellspecific and 39 of 43 differentially abundant miRNA were greater in abundance in CL. Interestingly, 93\% of the luteal-unique miRNA mapped to a $0.7 \mathrm{Mb}$ region of buffalo chromosome 20 (chromosome 21 of cows) and it was proposed that this miRNA cluster suppresses 20 alpha hydroxysteroid dehydrogenase, and thus progesterone metabolism, during luteinization (Baddela et al., 2017). In one study, a greater abundance of miRNA was found in follicular cells compared to luteal cells (Mohammed et al., 2017). Perhaps there is a robust expression of miRNA in developing and preovulatory follicles, that is then generally downregulated around the time of ovulation and early luteinization. Subsequent upregulation of miRNA during latter developmental stages of the CL would negatively regulate growth and support maximal steroidogenesis. It should be noted that in one recent study, there was no change in the number of miRNA in the midcycle compared to the early CL (Gecaj et al., 2017). It is unclear why this study differs from the previous ones.

Ma et al. (2011) compared nonregressed to regressed CL and reported $13 \mathrm{DE}$ miRNA, 7 being less abundant and 6 being more abundant in regressed CL. The most downregulated miRNA in regressed CL was miR378, and its expression appeared to be inversely correlated to its predicted target, IFNGR1 protein, suggesting that it may serve to repress IFNG-mediated cell death in the nonregressed CL. Using next generation sequencing, Maalouf et al. (2014) identified 544 known and 46 novel miRNA in the bovine CL. To determine if miRNA may be involved in luteal rescue during maternal recognition of pregnancy, CL collected on day 17 of the estrous cycle were compared to CL collected on the same day of pregnancy. Fifteen miRNA were found to be differentially abundant. The predicted targets of these 15 miRNA represent genes involved in immune-related events and apoptosis, reminiscent of pathways predicted to regulate luteal survival in the transcriptomic studies mentioned above. One of the miRNA targets associated with the top pathways in this study was CAMKK1, which, along with the mediator of acquisition of luteolytic capacity discussed previously, CAMKK2, plays a role in the calcium/calmodulindependent $(\mathrm{CaM})$ kinase cascade. This study indicated that miRNA are also likely to play in role in luteal rescue (Maalouf et al., 2014)

A comprehensive study of miRNA expression using miRNAseq has shown that some miRNA are highly abundant throughout luteal lifespan, whereas others are found in abundance only at specific stages (Gecaj et al., 2017). The dynamic and transitory nature of the CL makes it an exemplary tissue for demonstration of stage specificity of miRNA expression. The top 20 most abundant miRNA (based on mean reads from all stages of the cycle studied in each study) were identified for 
four miRNA profiling studies (Maalouf et al., 2014, 2016b, Baddela et al., 2017, Gecaj et al., 2017). MiRNA found in only one study, or common to two, three, or all four studies are listed in Table 1. The five miRNA common to at least three of these studies were analyzed in mirPath version 3 (Vlachos et al., 2015) and the top 10 gene ontology (GO) terms associated $\left(\mathrm{P}<10^{\wedge}-325\right)$ with their predicted targets, using the Tarbase database, are listed in Table 2. The top $10 \mathrm{GO}$ terms indicate that the most abundant miRNA in the CL are likely to be involved in regulation of the cell cycle, protein synthesis, and immune function. Notably, all five miRNA have predicted targets associated with each of the top $10 \mathrm{GO}$ terms, demonstrating possible redundancy in miRNA functions.

Finding significant differential expression of miRNA using profiling techniques may be affected by sample size, statistical analyses, isomiR distribution, relative abundance, and degree of variation among biological replicates. Thus, it is not surprising when some discrepancies in lists of DE miRNA in different studies occur. Researchers must use caution when drawing definitive biological conclusions based on somewhat arbitrary cutoffs for significance and variation in how data are handled.

Although profiling technologies have significantly enhanced understanding of miRNA in the corpus luteum, the targeted approaches that have sprung from these miRNA profiling studies have yielded important functional information about specific miRNA in the CL. Dai et al. (2014) reported an increase in the abundance of miR126 during luteal development, during which time its expression was inversely correlated to Talin2, suggesting that miR126 may regulate cellular interactions with extracellular matrix during final maturation of the CL. Maalouf et al. (2016b) also observed greater abundance of miR126 in midcycle compared to developing CL. miR96 is upregulated in the early CL compared to the follicle and supports survival of luteal cells by directly targeting FOXO1 (Mohammed et al., 2017). Angiogenesis in the developing $\mathrm{CL}$ is at least partially regulated by miR221 targeting thrombospondin 1 in luteal endothelial cells (Farberov and Meidan, 2017).

Table 1. miRNA identified among the top 20 most abundant miRNA in at least one of four miRNA profiling studies.

\begin{tabular}{ll}
\hline Number of studies & miRNA \\
\hline Four & let-7a-5p \\
Three & mir-21-5p, let-7f, mir-26a, let-7b \\
Two & let-7c, let-7d, let-7e, let-7g, let-7i, mir-100, mir-103, mir-10b, mir-125b, mir-143, \\
& mir-148a, mir-202, mir-30d, mir-320a, mir-3600 \\
One & let-7j, mir-107, mir-126-3p, mir-126-5p, mir-127, mir-140, mir-145. mir-148b, mir- \\
& $151-3 \mathrm{p}$, mir-154c, mir-1839, mir-186, mir-199a-3p, mir-214, mir-2284x, mir-23b, \\
& mir-24a, mir-26c, mir-27b, mir-29a, mir-30a-5p, mir-30e-5p, mir-320b, mir-320c, \\
& mir-342, mir-378, mir-423-5p, mir-450a, mir-486, mir-503-5p, mir-99a-5p, mir-99b \\
\hline
\end{tabular}

Table 2. Top 10 gene ontology (GO) terms associated with predicted targets of the 5 miRNA common to at least 3 of the studies.

\begin{tabular}{lc}
\hline GO Category & $\begin{array}{c}\text { Number of predicted target genes } \\
\text { associated with GO category }\end{array}$ \\
\hline G1/S transition of mitotic cell cycle & 69 \\
G2/M transition of mitotic cell cycle & 64 \\
Mitotic cell cycle & 188 \\
Protein binding transcription factor activity & 199 \\
Nucleic acid binding transcription factor activity & 292 \\
Toll-like receptor signaling pathway & 51 \\
Immune system process & 430 \\
MyD88-independent toll-like receptor signaling pathway & 42 \\
Molecular function & 4339 \\
RNA binding & 702 \\
\hline
\end{tabular}

\section{Proteomic profiling in the corpus luteum}

Inverse correlation of miRNA and mRNA abundance is often used as evidence that a particular mRNA is a target of a miRNA of interest. However, it is not always the case that a miRNA-mRNA interaction results in degradation of the target mRNA. If instead, the interaction simply causes translational repression of the mRNA, the steady state concentration of the mRNA may remain unchanged, or may increase if expression of the gene continues. Therefore, confirmation of a miRNA target requires determination of a change in protein concentration when the miRNA concentration is altered. An example of this is NOTCH1 regulation by miR34a in the CL (Fig. 3). Both miR34a and NOTCH1 mRNA are greater in the midcycle than the developing $\mathrm{CL}$, but the increase in NOTCH1 is not reflected by an increase in NOTCH1 protein, indicating either translational repression or enhanced protein degradation. In cultured luteal cells, a miR34a mimic clearly decreased NOTCH1, confirming translational repression by this miRNA. Therefore, global investigation of miRNA targets will be more reliable using proteomic, rather than transcriptomic, analyses. 
A.



C.

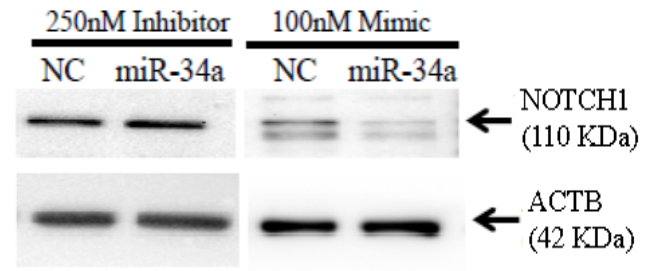

B.



D.



Figure 3. Relative expression of NOTCH1 (mRNA, A) and NOTCH1 (protein, B) in developing (day 4) and fully functional (MC=midcycle, days 10-12) CL. C) Representative western blot depicting downregulation of NOTCH1 in response to a miR-34a mimic compared to a negative control (NC) scrambled sequence RNA, and D) Mean ( $\mathrm{n}=$ 3) NOTCH1 protein abundance in response to miR-34a mimic. Adapted with permission from Maalouf et al. (2016b).

Few proteomics studies of the CL have been conducted and proteomic studies present challenges in terms of sensitivity. Often, identified proteins represent only the most highly abundant fraction of total proteins in a tissue. The two published studies of proteomics in the ruminant $\mathrm{CL}$ used two-dimensional polyacrylamide gel electrophoresis and MALDI-TOF-MS to identify proteins that changed during the estrous cycle or pregnancy. Arianmanesh et al. (2011) found that 139 proteins were upregulated and 69 were downregulated in the ovine $\mathrm{CL}$ as it progressed from day 12 to day 16 of the cycle. On day 16, plasma progesterone was low, indicating that the CL was regressed, but upregulated proteins included those involved in signal transduction, oxidative stress and structural integrity, indicating that the events of luteolysis are coordinated to induce cell death without a massive inflammatory response, rather than simply being a cessation of all cellular functions. In the progression from day 12 to day 16 of pregnancy, 52 proteins were upregulated and 14 were downregulated, suggesting that the presence of an embryo suppressed the changes in protein abundance that were apparent when the CL regressed. Upregulated proteins during pregnancy are involved in signal transduction, protein synthesis, electron transfer, steroidogenesis, and cytokine signaling (Arianmanesh et al., 2011).
Chung et al. (2012) compared the CL of day 90 of pregnancy to midcycle (days 6-13) CL from nonbred cattle. Analysis of the 2D gels revealed differences in protein abundance represented by 32 spots, and from these, 23 proteins were identified, of which 6 were more abundant and 17 were less abundant in CL of pregnancy. Differences in proportions of up- and downregulated proteins in these two studies are likely due to the different stages from which the CL of pregnancy were collected.

We have also used this procedure to compare bovine CL collected on day 18 of the cycle or pregnancy. The number of differentially expressed proteins was undetermined, but 18 spots that were clearly different in CL from cyclic or pregnant cattle (Fig. 4) were sequenced. Identified differentially abundant proteins included vimentin, adrenodoxin, 3hydroxymethylglutaryl-CoA synthase, apolipoprotein $\mathrm{A} 1$, annexin and glutathione S-transferase. There was considerable similarity in identified proteins between this study and the previous two. Six proteins were differentially abundant in at least two of these three proteomics studies (Arianmanesh et al., 2011; Chung et al., 2012; Pate et al., 2018; Penn State University, Center for Reproductive Biology and Health, University Park, PA USA; unpublished data). Commonly identified proteins among at least two of these three studies are 
listed in Table 3. Gene family was assigned by Ingenuity Pathway Analysis (IPA; Qiagen). The Diseases and Functions feature of IPA was used to identify functions associated with the commonly differentially abundant proteins. All significant $(\mathrm{P}<0.005)$ functions involving all six differentially abundant proteins and relevant functions involving four or five differentially abundant proteins are shown in Table 4. This analysis demonstrates that proteins that are modulated in the CL during early pregnancy are likely involved in regulating apoptosis and cell survival and maintaining steroidogenesis. Interestingly, all six common proteins were predicted to be involved in synthesis of lipid, which can be addressed in metabolomic studies.

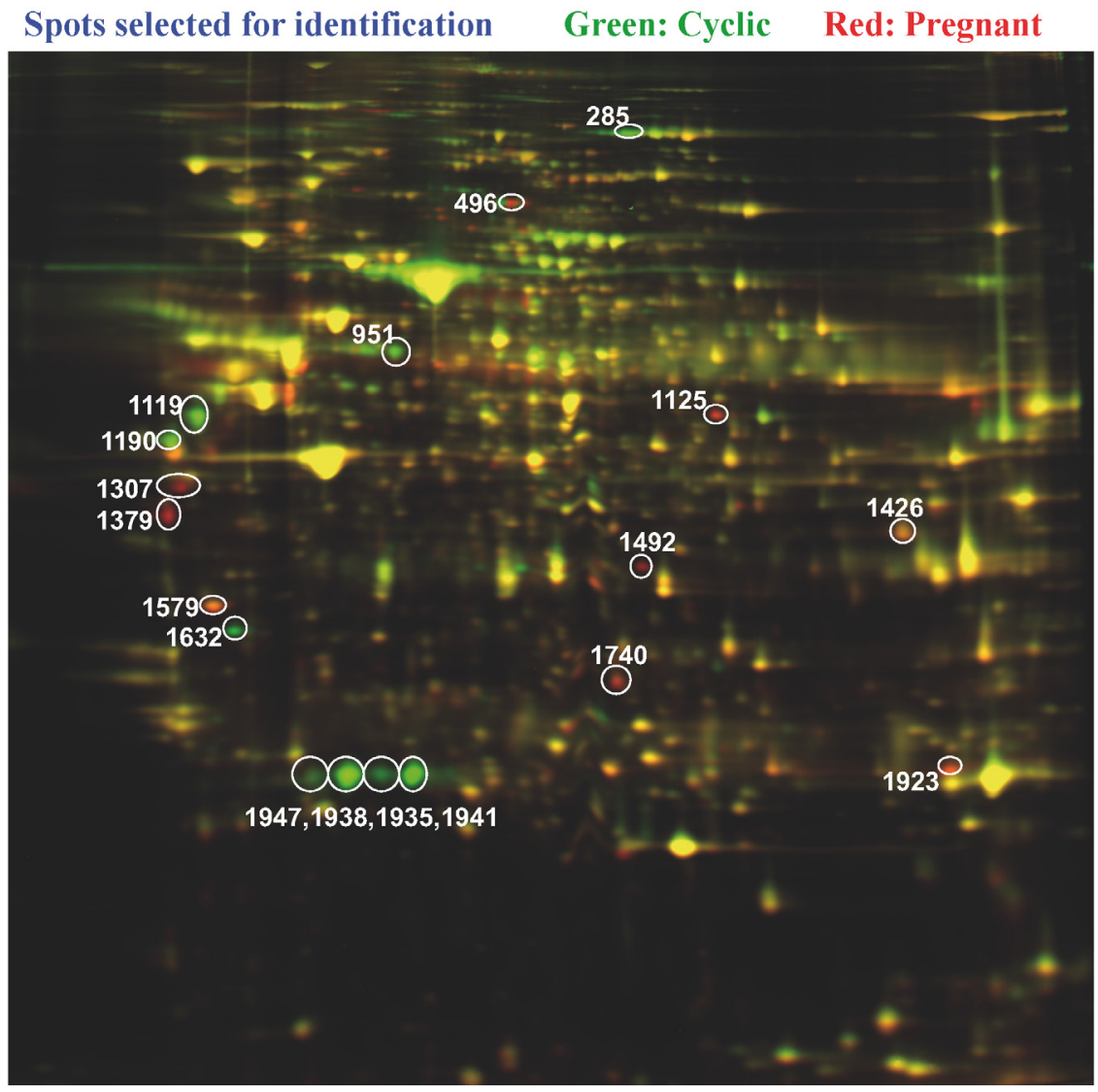

Figure 4. Representative 2D gel of proteins from CL collected on day 18 of the estrous cycle (green dye) and day 18 of pregnancy (red dye). Yellow indicates proteins that were of similar abundance in both treatment groups.

Table 3. Proteins identified as differentially abundant during the estrous cycle and pregnancy in at least two proteomics studies.

\begin{tabular}{lll}
\hline Protein & Gene symbol & Gene family \\
\hline Vimentin & VIM & other \\
Apolipoprotein A1 & APOA1 & transporter \\
Annexin $(5$ or A1) & ANXA1, ANXA5 & enzyme \\
Adrenodoxin reductase & FDXR & enzyme \\
Glutathione S-transferase & GSTA1 & enzyme \\
Superoxide dismutase & SOD1 & enzyme \\
\hline
\end{tabular}

Table 4: Functional analysis of proteins differentially abundant in the estrous cycle and pregnancy in at least two proteomics studies

\begin{tabular}{ll}
\hline Functions involving all 6 common proteins & Apoptosis, necrosis, synthesis of lipid \\
\hline $\begin{array}{l}\text { Relevant functions involving } 4 \text { or } 5 \text { common } \\
\text { proteins }\end{array}$ & $\begin{array}{l}\text { Fatty acid metabolism, migration of cells, synthesis of reactive } \\
\text { oxygen species, synthesis of steroid hormone, vasculogenesis }\end{array}$ \\
\hline
\end{tabular}




\section{Metabolomic studies of the corpus luteum}

Metabolomics is a broad term that can refer to measurement of metabolites of any biochemical processes, including amino acids, sugars, and lipids. Changes in specific lipid metabolites, such as prostaglandins, phospholipids, and steroidal molecules, as the CL progresses from development through luteolysis have been achieved using various targeted approaches, such as chromatography and radioimmunoassay. However, no comprehensive metabolomic study of the CL has been reported. We have recently completed a comprehensive analysis of lipid metabolite concentrations in the CL during the estrous cycle, luteolysis and maternal recognition of pregnancy (Hughes et al., 2018; Penn State University, Center for Reproductive Biology and Health, University Park, PA USA; unpublished data). Among 79 lipids measured, there were 24 lipids that differed in abundance during the estrous cycle, all being less abundant on day 4 than on day 11 , with nine remaining high on day 18 of the cycle. During a 24-h time-course of luteolysis, 35 lipids changed, and as might be expected, represented arachidonic acid metabolism and prostaglandin signaling. In the early period of maternal recognition of pregnancy, only subtle changes in mRNA, miRNA and proteins are detectable in the $\mathrm{CL}$, and this was reflected in the metabolic profile of the bovine CL on day 18 .

While there are many programs available for functional and pathway analysis of transcriptomics and proteomics data, fewer such programs are available for analysis of metabolomic data and integration of metabolomic and transcriptomic data. Using the data integration feature in the program MetaboAnalyst (Xia and Wishart, 2016), pathways including sphingolipid (ceramide) metabolism, propanoate metabolism, and pyruvate metabolism were indicated as differentially regulated in the CL of pregnancy. However, close examination of these results revealed that these pathways were modulated by either genes or lipids, but not both, indicating that this program resulted in a listing of potential metabolic pathway modulation, without lipid-metabolite integration. In IPA, the Network and Diseases and Functions features were much more useful in demonstrating functions that may be regulated by combinations of differentially abundant genes and lipids. The top networks containing both differentially abundant genes and lipids were Lipid metabolism, molecular transport, and small molecule biochemistry and DNA replication, recombination and repair, cell death and survival, cellular function and maintenance. Further, differentially abundant mRNA and lipids were expected to be involved in cell movement and migration; among significant diseases or functions annotations, these pathways included the greatest total number of molecules, including both mRNA and lipids (Fig. 5). Other pathways were related to cell interaction and to immune cell differentiation.

\section{Conclusion}

Although large-scale molecular profiling studies have largely supported existing hypotheses about luteal function, they have also allowed identification of novel signaling pathways that could be targeted to support luteal function. In particular, these studies have contributed to our understanding of the CL of pregnancy, which, because of the subtlety of the changes that occur in these CL, have been difficult to study using targeted approaches in the past. Figure 6 shows a summary of what large-scale molecular profiling and pathway analysis has revealed about functions that are modulated in the CL during early pregnancy. Notably, each of these technologies, except proteomics, have indicated that luteal immune cell function may be modulated during early pregnancy (with proteomics suggesting cell migration, which is likely migration of immune cells), a compelling finding in light of the growing body of evidence that immune cells are intricately involved in regulation of luteal functions.

Application of large-scale molecular profiling to study the corpus luteum is still in a nascent stage, and laboratory experiments to confirm functions suggested by these kinds of studies are necessary to elucidate specific regulation of luteal function. However, improvements in statistical analysis techniques, as well as advances in the profiling technologies themselves, to improve accuracy and precision with which miRNA, mRNA, proteins, and metabolites can be measured, will continue to drive these kinds of studies forward and allow generation of high-quality, high-resolution data, to more completely understand luteal function. 
Cell movement 1

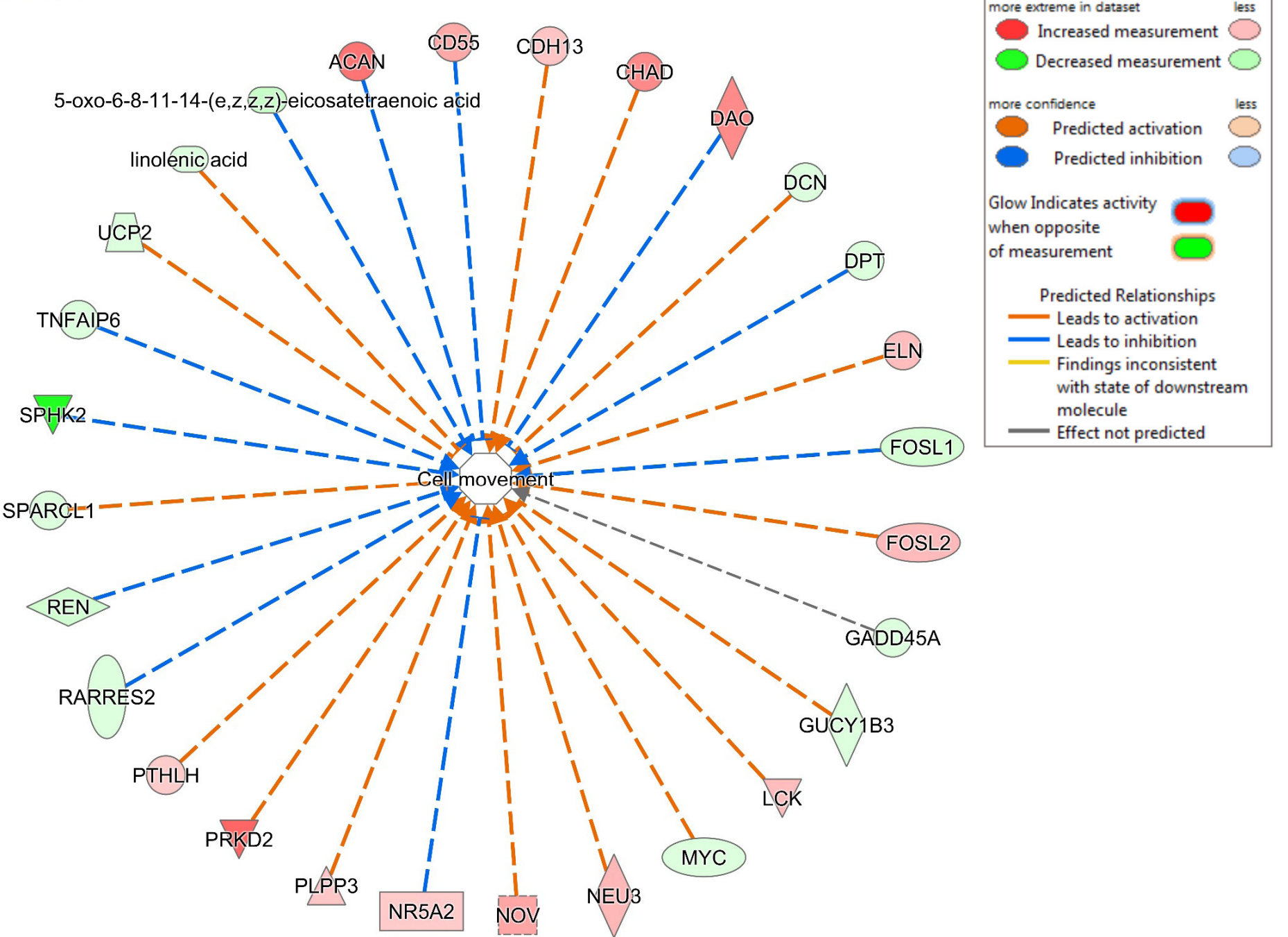

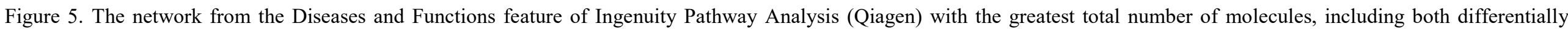

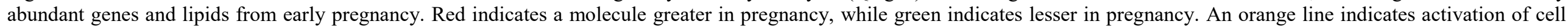
movement by a molecule, while a blue line indicates inhibition of cell movement. 


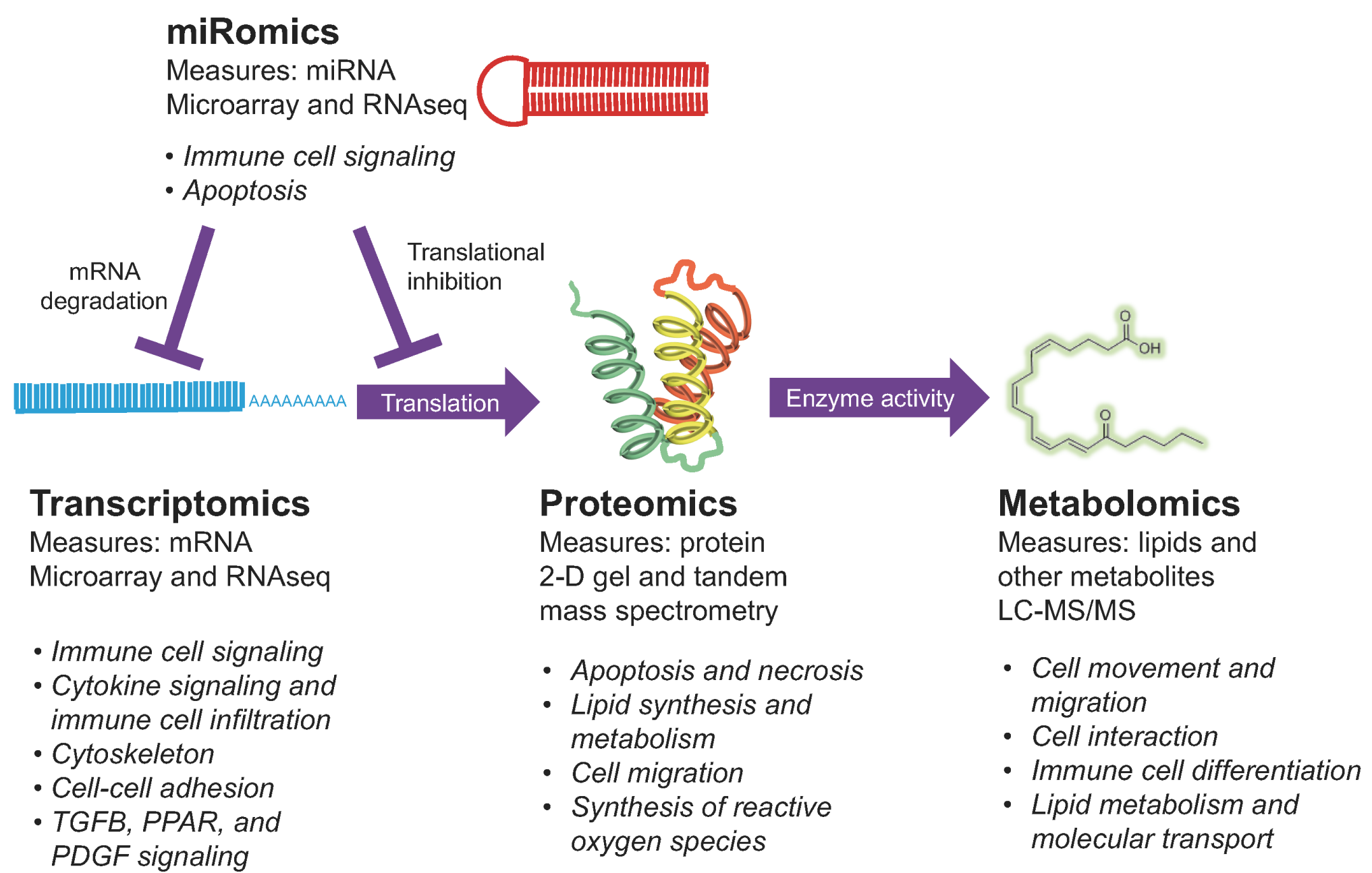

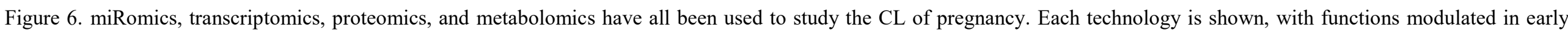

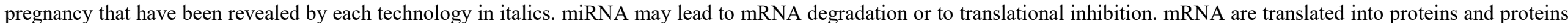
mediate the production of lipids and other metabolites that may have key signaling functions. 


\section{References}

Alila HW, Hansel W. 1984. Origin of different cell types in the bovine corpus luteum as characterized by specific monoclonal antibodies. Biol Reprod, 31:10151025.

Arianmanesh M, McIntosh RH, Lea RG, Fowler PA, Al-Gubory KH. 2011. Ovine corpus luteum proteins, with functions including oxidative stress and lipid metabolism, show complex alterations during implantation. $J$ Endocrinol, 210:47-58.

Atli MO, Bender RW, Mehta V, Bastos MR, Luo W, Vezina CM, Wiltbank MC. 2012. Patterns of gene expression in the bovine corpus luteum following repeated intrauterine infusions of low doses of prostaglandin F2alpha. Biol Reprod, 86:130. doi: 10.1095/biolreprod.111.094870.

Baddela VS, Onteru SK, Singh D. 2017. A syntenic locus on buffalo chromosome 20: novel genomic hotspot for miRNAs involved in follicular-luteal transition. Funct Integr Genomics, 17:321-334.

Baddela VS, Koczan D, ViergutzT, Vernunft A, Vanselow J. 2018. Global gene expression analysis indicates that small luteal cells are involved in extracellular matrix modulation and immune cell recruitment in the bovine corpus luteum. Mol Cell Endocrinol. doi: 10.1016/j.mce.2018.03.011.

Benjamini Y, Hochberg Y. 1995. Controling the false discovery rate: a practical and powerful approach to multiple testing. J Royal Stat Soc, 57:289-300.

Bowdridge EC, Goravanahally MP, Inskeep EK, Flores JA. 2015. Activation of adenosine monophosphate-activated protein kinase is an additional mechanism that participates in mediating inhibitory actions of prostaglandin F2alpha in mature, but not developing, bovine corpora lutea. Biol Reprod, 93:7. doi: 10.1095/biolreprod.115.129411.

Catalanotto C, Cogoni C, Zardo G. 2016. MicroRNA in control of gene expression: an overview of nuclear functions. Int $J$ Mol Sci, 17(10): pii: E1712. doi: 10.3390/ijms17101712.

Christenson LK. 2010. MicroRNA control of ovarian function. Anim Reprod, 7:129-133.

Chung HJ, Kim KW, Han DW, Lee HC, Yang BC, Chung HK, Shim MR, Choi MS, Jo EB, Jo YM, Oh MY, Jo SJ, Hong SK, Park JK, Chang WK. 2012. Protein profile in corpus luteum during pregnancy in Korean native cows. Asian-Aust J Anim Sci, 25:15401545 .

Farberov S, Meidan R. 2017. Fibroblast growth factor2 and transforming growth factor-beta1 oppositely regulate miR-221 that targets thrombospondin-1 in bovine luteal endothelial cells. Biol Reprod, 98:366375 .

Fatima LA, Baruselli PS, Gimenes LU, Binelli M, Renno FP, Murphy BD, Papa PC. 2012. Global gene expression in the bovine corpus luteum is altered after stimulatory and superovulatory treatments. Reprod Fertil Dev, 25:998-1011.

Frobenius W. Ludwig Fraenkel: Spiritus rector of the early progesterone research. Eur J Obstet Gynecol Reprod Biol, 83:115-119.
Gecaj RM, Schanzenbach CI, Kirchner B, Pfaffl MW, Riedmaier I, Tweedie-Cullen RY, Berisha B. 2017. The dynamics of microRNA tramscriptome in bovine corpus luteum during its formation, functoin, and regression. Front Genet, 8:213. doi: 10.3389/fgene.2017.00213.

Goravanahally MP, Salem M, Yao J, Inskeep EK, Flores JA. 2009. Differential gene expression in the bovine corpus luteum during transition from early phase to midphase and its potential role in acquisition of luteolytic sensitivity to prostaglandin F2 alpha. Biol Reprod, 80:980-988.

He L, Hannon GJ. 2004. MicroRNAs: Small RNAs with a big role in gene regulation. Nature Rev Genetics, 5:522-531.

Hossain MM, Ghanem N, Hoelker M, Rings F, Phatsara C, tholen E, Schelander K, Tesfaye D. 2009. Identification and characterization of miRNAs expressed in the bovine ovary. BMC Genomics, 10:443. doi:10.1186/1471-2164-10-443.

Hossain MM, Sohel MM, Schellander K, Tesfaye D. 2012. Characterization and importance of microRNAs in mammalian gonadal functions. Cell Tissue Res, 349:679-690.

Jocelyn HD, Setchell BP. 1972. Regnier deGraaf on the human reproductive organs: an annotated translation of tractatus de virirum organis generationi inservientibus (1668) and de mulierum organis generationi servientibus tractatus novus (1672). $J$ Reprod Fertil Suppl, 17:1-222.

Kfir S, Basavaraja R, Wigoda N, Ben-Dor S, Orr I, Meidan R. 2018. Genomic profiling of bovine corpus luteum maturation. PLoS One, 13(3):e0194456. doi: 10.1371/journal.pone.0194456.

Li Y, Fang Y, Liu Y, Yang X. 2015. MicroRNAs in ovarian function and disorders. J Ovarian Res, 8:51-58.

Ma T, Jiang H, Gao Y, Zhao Y, Dai L, Xiong Q, Xu Y, Zhao Z, Zhang J. 2011. Microarray analysis of differentially expressed microRNAs in nonregressed and regressed bovine corpus luteum tissue; microRNA378 may suppress luteal cell apoptosis by targeting the interferon gamma receptor 1 gene. $J$ Appl Genet, 52:481-486

Maalouf SW, Liu W-S, Albert I, Pate, JL. 2014. Regulating life or death: potential role of microRNA in rescue of the corpus luteum. Mol Cell Endocrinol, 398:78-88.

Maalouf SW, Liu WS, Pate JL. 2016a. MicroRNA in ovarian function. Cell Tissue Res, 363:7-18.

Maalouf SW, Smith CL, Pate JL. 2016b. Changes in microRNA expression during maturation of the bovine corpus luteum: regulation of luteal cell proliferation and function by microRNA-34a. Biol Reprod, 94:71. doi: 10.1095/biolreprod.115.135053.

McBride D, Carré W, Sontakke SD, Hogg CO, Law A, Donadeu FX, Clinton M. 2012. Reproduction, 144:221-233

McGinnis LK, Luense LJ, Christenson LK. 2015. MicroRNA in ovarian biology and disease. Cold Spring Harb Perspect Med, 5(9):a022962. doi: 10.1101/cshperspect.a022962.

Miyamoto A, Shirasuna K, Shimizu T, Matsui M. 
2013. Impact of angiogenic and innate immune systems on the corpus luteum function during its formation and maintenance in ruminants. Reprod Biol, 13:272-278.

Mohammed BT, Sontakke SD, Ioannidis J, Duncan WC, Donadeu FX. 2017. The adequate corpus luteum: miR-96 promotes luteal cell survival and progesterone production. J Clin Endocrimol Metab, 102:2188-2198.

Mondal M, Schilling B, Folger J, Steibel JP, Buchnick H, Zalman Y, Ireland JJ, Meidan R, Smith GW. 2011. Deciphering the luteal transcriptome: potential mechanisms mediating stage-specific luteolytic response of the corpus luteum to prostaglandin F2 $\alpha$. Physiol Genomics, 43:447-456.

Moore SG, Pryce JE, Hayes BJ, Chamberlain AJ, Kemper KE, Berry DP, McCabe M, Cormican P, Lonergan P, Fair T, Butler ST. 2016. Differentially expressed genes in endometrium and corpus luteum of holstein cows selected for high and low fertility are enriched for sequence variants associated with fertility. Biol Reprod, 94:19. doi 10.1095/biolreprod.115.132951.

Mudge JF, Martyniuk CJ, and Houlahan JE. 2017 Optimal alpha reduces error rates in gene expression studies: a meta-analysis approach. BMC Bioinformatics, 18:312. doi: 10.1186/s12859-017-1728-3.

Niswender GD, Juengel JL, Silva PJ, Rollyson MK, McIntush EW. 2000. Mechanisms controlling the function and life span of the corpus luteum. Physiol Rev, 80:1-29. doi: 10.1152/physrev.2000.80.1.1

Ochoa JC, Peñagaricanoc F, Baez GM, Melo LF, Motta JC, Guerra AG, Meidan R, Ferreira JCP, Sartori R, Wiltbank MC. 2018. Mechanisms for rescue of CL during pregnancy: gene expression in bovine $\mathrm{CL}$ following intrauterine pulses of prostaglandins $\mathrm{E} 1$ and $\mathrm{F} 2 \alpha$. Biol Reprod. doi: 10.1093/biolre/iox183.

Pate JL, Johnson-Larson CJ, Ottobre JS. 2012. Life or death decisions in the corpus luteum. Reprod Domest Anim, 47(suppl. 4):297-303.

Romereim SM, Summer AF, Pohlmeier WE, Zhang P, Hou X, Talbott HA, Cushman RA, Wood JR, Davis JS, Cupp AS. 2017. Gene expression profiling of bovine ovarian follicular and luteal cells provides insight into cellular identities and functions. Mol Cell Endocrinol, 439:379-394.

Romero JJ, Antoniazzi AQ, Smirnova NP, Webb BT, Yu F, Davis JS, Hansen TR. 2013. Pregnancyassociated genes contribute to antiluteolytic mechanisms in ovine corpus luteum. Physiol Genomics, 45:1095-1108.

Sakumoto R, Hayashi K-G, Hosoe M, Iga, K, Kizaki K, Okuda K. 2015. Gene expression profiles in the bovine corpus luteum (CL) during the estrous cycle and pregnancy: Possible roles of chemokines in regulating CL function during pregnancy. J Reprod Dev, 61:42-48. Shah KB, Tripathy S, Suganthi H, Rudraiah M.
2014. Profiling of luteal transcriptome during prostaglandin F2-alpha treatment in buffalo cows: analysis of signaling pathways associated with luteolysis. PLOS One, 9(8):e104127. doi:10.1371/journal.pone.0104127.

Simmer HH. 1971. The first experiments to demonstrate an endocrine function of the corpus luteum on the occasion of the 100 th birthday of Ludwig Fraenkel (1870-1951). Sudhoffs Arch, 55:392-417.

Smith GW, Meidan R. 2014. Ever-changing cell interactions during the lifespan of the corpus luteum: relevance to luteal regression. Reprod Biol, 14:75-82.

Talbott H, Hou X, Qiu F, Zhang P, Guda C, Yu F, Cushman RA, Wood JR, Wang C, Cupp AS, Davis JS. 2017. Early transcriptome responses of the bovine midcycle corpus luteum to prostaglandin F2 $\alpha$ includes cytokine signaling. Mol Cell Endocrinol, 452:93-109.

Tesfaye D, Gebremedhn S, Salilew-Wondim D, Hailay T, Hoelker M, Grosse-Brinkhaus C, Schellander K. 2018. MicroRNAs: tiny molecules with a significant role in mammalian follicular and oocyte development. Reproduction, 155:R121-R135. Treiber T, Treiber N, Meister G. 2012. Regulation of microRNA biogenesis and function. Thromb Haemost, 107:605-610.

Tsai S-J, Wiltbank MC. 1998. Prostaglandin F2 $\alpha$ regulates distinct physiological changes in early and midcycle bovine corpora lutea. Biol Reprod, 58:346352.

Villa-Godoy A, Ireland JJ, Wortman JA, Ames NK, Highes TL, Fogwell RL. 1985. Effect of ovarian follicles on luteal regression in heifers. J Anim Sci, 60:519-527.

Vlachos IS, Zagganas K, Paraskevopoulou MD, Georgakilas G, Karagkouni D, Vergoulis T, Dalamagas T, Hatzigeorgiou AG. 2015. DIANAmiRPath v3. 0: deciphering microRNA function with experimental support. Nucleic Acids Res, 43:460-466.

Wiltbank JN, Ingalls JE, Rowden WW. 1961. Effects of various forms and levels of estrogens alone or in combination with gonadotropins on the estrous cycle of beef heifers. J Anim Sci, 20:341-346

Wiltbank MC, Salih SM, Atli MO, Luo W, Bormann CL, Ottobre JS, Vezina CM, Mehta V, Diaz FJ, Tsai SJ, Sartori R. 2012. Comparison of endocrine and cellular mechanisms regulating the corpus luteum of primates and ruminants. Anim Reprod, 9:242-259.

Xia J, Wishart DS. 2016. Using metaboanalyst 3.0 for comprehensive metabolomics data analysis. Curr Protoc Bioinformatics, 55:14.10.1-14.10.91

Zalman Y, Klipper E, Farberov S, Mondal M, Wee G, Folger JK, Smith GW, Meidan R. 2012. Regulation of angiogenesis-related prostaglandin F2alpha-induced genes in the bovine corpus luteum. Biol Reprod, 86:92. doi: 10.1095/biolreprod. 111.095067. 